(1)

CrossMark

\section{Ambient ozone and influenza transmissibility in Hong Kong}

\author{
To the Editor:
}

Understanding the environmental drivers of influenza transmissibility would contribute to the early intervention and long-term control strategies of seasonal influenza, a serious public health problem that causes considerable morbidity and mortality each year. Within the burgeoning literature on influenza transmission, there are conflicting lines of evidence on the role of the environment [1]. Besides meteorological factors, it is also uncertain how common air pollutants such as ozone $\left(\mathrm{O}_{3}\right)$, sulfur dioxides $\left(\mathrm{SO}_{2}\right)$, nitrogen dioxide $\left(\mathrm{NO}_{2}\right)$, nitric oxide $(\mathrm{NO})$ and particulate matter may affect influenza transmission [2]. The objective of our study was to examine the relationship of influenza transmissibility in Hong Kong with common air pollutants and other environmental factors, including ultraviolet radiation and absolute humidity.

A number of earlier studies on the environmental drivers of influenza transmission used absolute counts of influenza cases as the dependent variable in statistical models. However, the number of incident influenza cases is not an ideal representation of influenza transmission intensity [3]. We estimated the daily effective reproduction number $(R t)$, a real-time measure of transmissibility, for each influenza type/ subtype using data from the subtropical city of Hong Kong, which has excellent influenza surveillance data, near year-round circulation of influenza, and considerable variations in environmental factors and pollutant levels. We combined information on influenza-like illnesses (ILIs) in the community and laboratory surveillance data to estimate weekly incidence rates of influenza virus infections in the community, referred to as $\mathrm{ILI}^{+}$rates [4]. In theory, this time series should be a linear correlate of the incidence rate of infections in the community [4], and it was previously shown that there was a very close correlation between this measure and laboratory confirmed H1N1pdm09 hospitalisations in Hong Kong in 2009-2010 [5]. Finally, we multiplied the weekly $\mathrm{ILI}^{+}$rates by a large constant, representing the inverse of the coverage of the sentinel sites in Hong Kong, and rounded to the nearest integer to obtain a time series of weekly $\mathrm{ILI}^{+}$counts (figure 1a-d). This was then interpolated to daily $\mathrm{ILI}^{+}$counts using splines. During the study period of January 1998 to December 2013, we identified 44 distinct influenza epidemics, including 16 epidemics of seasonal influenza $\mathrm{A}(\mathrm{H} 3 \mathrm{~N} 2), 10$ of $\mathrm{A}(\mathrm{H} 1 \mathrm{~N} 1)$, four of $\mathrm{A}(\mathrm{H} 1 \mathrm{~N} 1 \mathrm{pdm} 09)$ and 14 of influenza B (figure 1a-d). Daily concentrations of major air pollutants in 10 local monitoring stations were used to calculate the territory-wide daily average concentrations for Hong Kong. Meteorological data were obtained from the Hong Kong Observatory.

Transmissibility can be measured by the effective (or instantaneous) $R t$ as a unit-free index of outbreak intensity, defined as the average number of secondary infections caused in the population by a typical single infectious person at time $t$. We estimated $R t$ from daily $\mathrm{ILI}^{+}$counts for each influenza type/subtype. We adopted a simple branching process model [6] to estimate daily $R t$ values. We assumed a Gamma distribution for the serial interval with mean \pm SD values of $3.08 \pm 1.39$ for influenza A(H1N1pdm09), 3.26 \pm 1.93 for $\mathrm{A}(\mathrm{H} 1 \mathrm{~N} 1), 3.48 \pm 1.88$ for $\mathrm{A}(\mathrm{H} 3 \mathrm{~N} 2)$ and $3.72 \pm 1.95$ for influenza B [7].

We used regression models to explore the association between influenza transmissibility, measured by the daily estimated effective $R t$ for up to 8 weeks either side of each epidemic peak, and various pollutant factors with 0-7 days lag values. In nonlinear univariate regression analysis, we found that $R t$ had statistically significant negative association with ambient $\mathrm{O}_{3}$ across all the types/subtypes; $\mathrm{NO}$ and $\mathrm{CO}$ had a weak positive association with influenza transmissibility, while other pollutants had no consistent patterns and the estimated effects were generally not statistically significant. The estimated nonlinear effect of ozone on influenza transmissibility is shown in figure 1f. The multivariable regression (DLM, dlmn

@ERSpublications

Higher levels of ambient ozone are associated with reduced influenza transmissibility http://ow.ly/zr9030j3Tcn

Cite this article as: Ali ST, Wu P, Cauchemez S, et al. Ambient ozone and influenza transmissibility in Hong Kong. Eur Respir J 2018; 51: 1800369 [https://doi.org/10.1183/13993003.00369-2018]. 


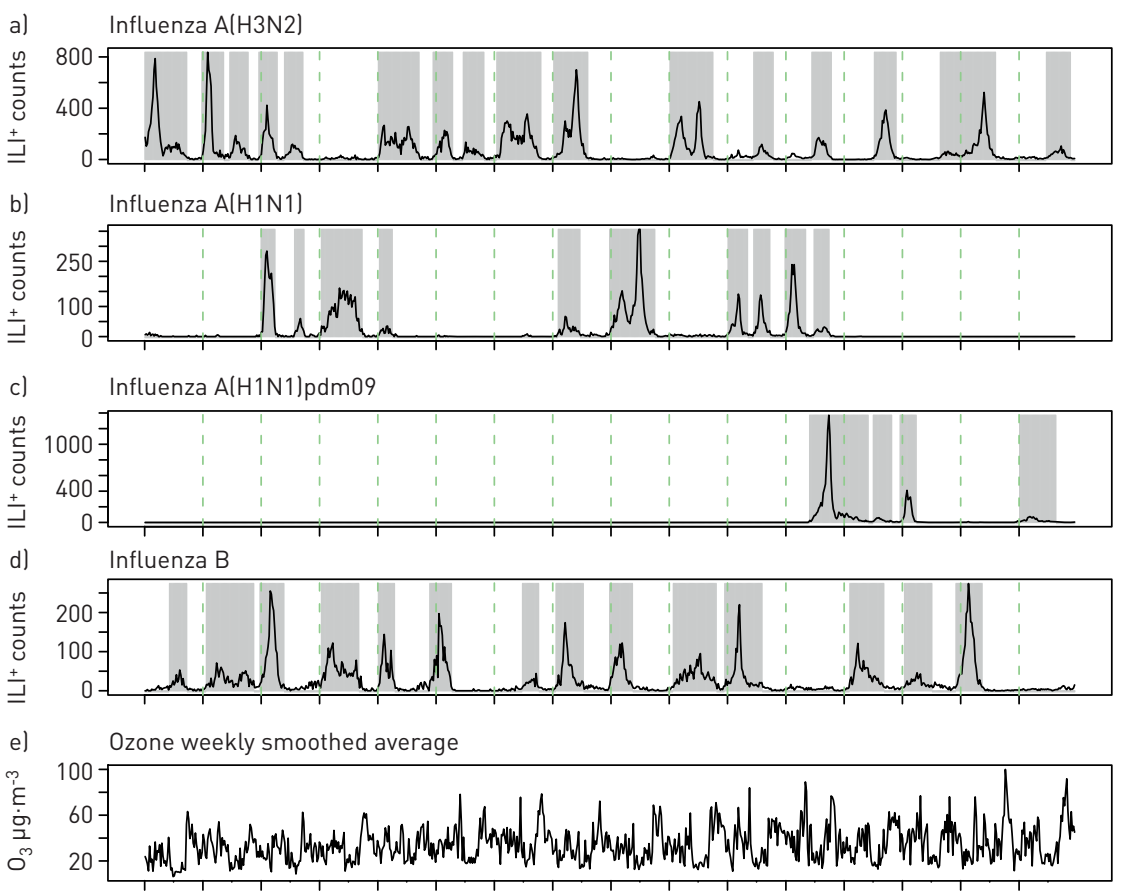

1998199920002001200220032004200520062007200820092010201120122013

f)

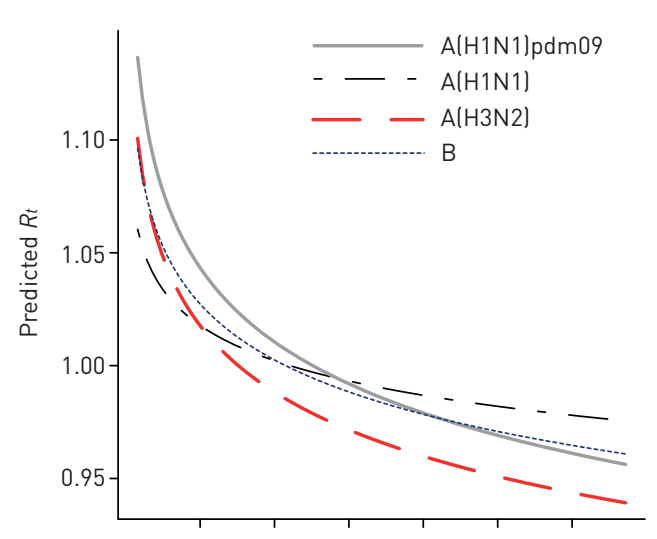

o

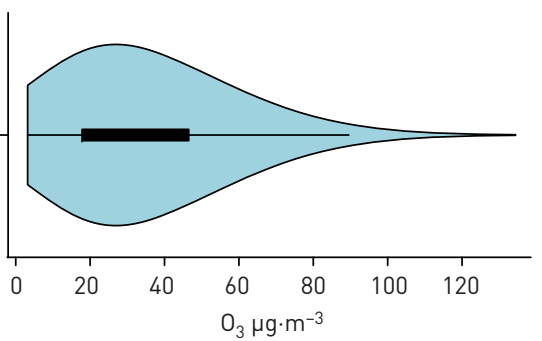

FIGURE 1 Weekly activity of influenza (influenza-like illness (ILI)+ proxy) of a) influenza A(H3N2), b) influenza A(H1N1), c) influenza A(H1N1)pdm09 and d) influenza B (black lines) along with the 44 predefined epidemics (grey bars) of these virus type and subtypes, and e) the weekly smoothed average of ozone $\left(\mathrm{O}_{3}\right)$ concentrations in Hong Kong from 1998 to 2013 . $f$ ) Estimated nonlinear relationship between the effective reproduction number $(R t)$ and ambient daily $\mathrm{O}_{3}$ concentrations in the regression analysis (based on the selected best-fitting lag of 5 days for $\mathrm{A}$ (H3N2), 6 days for $A(H 1 N 1), 7$ days for $A(H 1 N 1) p d m 09$ and 4 days for influenza B) for influenza A(H3N2), A(H1N1) prior to 2009, A(H1N1)pdm09 from 2009 onwards and influenza B. The violin plot shown in the lower panel indicates the distribution of daily ambient ozone concentrations; the median is indicated by the white circle, the interquartile range is indicated by the black rectangle and the blue area displays a kernel density estimate of the distribution of values (i.e. a smoothed histogram). 
package in R) model that included depletion of susceptibles, interepidemic factors, absolute humidity and ambient $\mathrm{O}_{3}$ could explain $40 \%$ of the observed variation in $R t$ for seasonal influenza $\mathrm{A}(\mathrm{H} 3 \mathrm{~N} 2), 35 \%$ for seasonal influenza $\mathrm{A}(\mathrm{H} 1 \mathrm{~N} 1), 60 \%$ for $\mathrm{A}(\mathrm{H} 1 \mathrm{~N} 1) \mathrm{pdm} 09$ and $21 \%$ for influenza $\mathrm{B}$. With a large proportion of the variance explained by the intrinsic factors and absolute humidity in the basic model for influenza transmissibility, the ambient $\mathrm{O}_{3}$ contributed only marginally, explaining a further $4 \%$ of the total variance in influenza transmissibility for $\mathrm{H} 3 \mathrm{~N} 2$ and up to $1 \%$ for the other three influenza types/subtypes. A permutation analysis indicated that the association was not likely to be due to chance (data not shown). While the proportion of variance in influenza transmissibility explained by $\mathrm{O}_{3}$ is modest, this could still correspond to a substantial effect on incidence in a single epidemic, which includes many transmission events [8]. In Hong Kong, seasonal influenza often exhibits twice-annual peaks in periods from July to August (summer) and from January to March (late winter/early spring), which generally coincide with two troughs of $\mathrm{O}_{3}$ concentration seasonality (figure 1a-e).

The association of ambient $\mathrm{O}_{3}$ with reduced influenza transmissibility may be related to the virucidal activity of $\mathrm{O}_{3}$ and its effect on host defense. $\mathrm{O}_{3}$ inactivation of influenza virus within a few hours has been reported in studies in vitro [9]. However, a more plausible mechanism underlying the association of $\mathrm{O}_{3}$ with a reduction in influenza transmissibility is $\mathrm{O}_{3}$-primed immunity against influenza virus infection. Inhalation of ambient $\mathrm{O}_{3}$ can enhance pulmonary innate immunity that promote allergic responses in healthy human subjects and susceptible populations [10]. It is not likely that $\mathrm{O}_{3}$ as an oxidant gas is directly recognised by a discrete receptor; $\mathrm{O}_{3}$-induced inflammation is probably mediated by a secondary messenger. One such candidate is interleukin (IL)-33. Induced by $\mathrm{O}_{3}$ exposure, IL-33 further activates type 2 cytokines in the lung. IL-33 appears to be the common denominator for the list of asthma triggers including allergy, viral infection and $\mathrm{O}_{3}$ [11]. As a multifaceted cytokine, however, IL-33 not only play a pathogenic role in Th2-mediated diseases but also drives Th1 and CD8 T-cell responses that induce protective immunity against viral infections [12]. In the case of influenza, IL-33 promotes lung tissue homeostasis during viral infection [13]. Used as an adjuvant in influenza vaccines, IL-33 increases the antigen-specific CD4 and CD8 T-cell responses in preclinical settings [14].

One limitation of the present study was the interpolation of daily $\mathrm{ILI}^{+}$counts from the weekly data. The day-to-day variation in transmissibility might have been reduced because of this interpolation, leading to underestimated effects of the drivers for influenza. If available, using $\mathrm{ILI}^{+}$data at a daily scale would improve the estimates. Another limitation is that the territory-wide daily average calculation might introduce measurement errors for certain pollutants such as $\mathrm{NO}_{2}$ and $\mathrm{CO}$, which have a relatively large spatial variability. However, if the spatial variability did not change systematically with time, the aggregated exposure measurement should not bias the study findings based on territory-wide time-series data of both influenza and environmental drivers.

As a highly reactive oxidant air pollutant, $\mathrm{O}_{3}$ may decrease host defences against bacterial and fungal infections in the airways, and aggravate pre-existing diseases such as asthma. In the case of influenza, however, ambient $\mathrm{O}_{3}$ had not been consistently associated with hospital admissions or emergency department visits for influenza virus infections according to the review by the US Environmental Protection Agency in 2013 [10]. Our current findings of reduced influenza transmissibility associated with ambient $\mathrm{O}_{3}$ in Hong Kong warrant further study.

Sheikh Taslim Ali ${ }^{1}$, Peng $\mathrm{Wu}^{1}$, Simon Cauchemez ${ }^{2,3,4}$, Daihai $\mathrm{He}^{5}$, Vicky J. Fang ${ }^{1}$, Benjamin J. Cowling ${ }^{1}$ and Linwei $\operatorname{Tian}^{1}$

${ }^{1}$ WHO Collaborating Centre for Infectious Disease Epidemiology and Control, School of Public Health, Li Ka Shing Faculty of Medicine, The University of Hong Kong, Hong Kong Special Administrative Region, China. ${ }^{2}$ Mathematical Modelling of Infectious Diseases Unit, Institut Pasteur, Paris, France. ${ }^{3}$ Centre National de la Recherche Scientifique, URA3012, Paris, France. ${ }^{4}$ Center of Bioinformatics, Biostatistics and Integrative Biology, Institut Pasteur, Paris, France. ${ }^{5}$ Dept of Applied Mathematics, Hong Kong Polytechnic University, Hong Kong Special Administrative Region, China.

Correspondence: Benjamin J. Cowling, School of Public Health, Li Ka Shing Faculty of Medicine, The University of Hong Kong, 7 Sassoon Road, Pokfulam, Hong Kong. E-mail: bcowling@hku.hk

Received: Oct 242017 | Accepted after revision: Feb 272018

Acknowledgments: The authors thank Julie Au (The University of Hong Kong, Hong Kong Special Administrative Region, China) for technical assistance.

Author contributions: B.J. Cowling and L. Tian designed the study. S.T. Ali, P. Wu, V.J. Fang and L. Tian collected the data. S.T. Ali analysed the data. S.T. Ali wrote the first draft of the manuscript, and all authors contributed to review and revision, and have seen and approved the final version.

Support statement: This work was financially supported by grants from the Health and Medical Research Fund, the National Institute of General Medical Sciences (grant number U54 GM088558) and the Hong Kong Research Grants 
Council (project number T11-705/14 N). D. He was supported by the Early Career Scheme from the Hong Kong Research Grants Council (PolyU 251001/14M). Funding information for this article has been deposited with the Crossref Funder Registry.

Conflict of interest: B.J. Cowling has received research funding from Sanofi Pasteur for a study of influenza vaccine effectiveness.

\section{References}

1 Sooryanarain H, Elankumaran S. Environmental role in influenza virus outbreaks. Annu Rev Anim Biosci 2015; 3 : 347-373.

2 Loveren HV, Rombout PJA, Fischer PH, et al. Modulation of host defenses by exposure to oxidant air pollutants. Inhal Toxicol 1995; 7: 405-423.

3 Te Beest DE, Van Boven M, Hooiveld M, et al. Driving factors of influenza transmission in the Netherlands. Am J Epidemiol 2013; 178: 1469-1477.

4 Goldstein E, Viboud C, Charu V, et al. Improving the estimation of influenza-related mortality over a seasonal baseline. Epidemiology 2012; 23: 829-838.

5 Wong JY, Wu P, Nishiura $\mathrm{H}$, et al. Brief original contribution infection fatality risk of the pandemic A (H1N1) 2009 virus in Hong Kong. Am J Epidemiol 2013; 177: 834-840.

6 Cori A, Ferguson NM, Fraser C, et al. A new framework and software to estimate time-varying reproduction numbers during epidemics. Am J Epidemiol 2013; 178: 1505-1512.

7 Levy JW, Cowling BJ, Simmerman JM, et al. The serial intervals of seasonal and pandemic influenza viruses in households in Bangkok, Thailand. Am J Epidemiol 2013; 177: 1443-1451.

8 Dushoff J, Plotkin JB, Levin SA, et al. Dynamical resonance can account for seasonality of influenza epidemics. Proc Natl Acad Sci 2004; 101: 16915-16916.

9 Tseng C-C, Li C-S. Ozone for inactivation of aerosolized bacteriophages. Aerosol Sci Technol 2006; 40: 683-689.

10 USEPA. Integrated science assessment for ozone and related photochemical oxidants. Fed Regist 2013; 78: 11172-11173.

11 Mathews J, Krishnamoorthy N, Kasahara DI, et al. IL-33 drives augmented responses to ozone in obese mice. Environ Health Perspect 2016; 125: 246-253.

12 Bonilla W V, Fröhlich A, Senn K, et al. The alarmin interleukin-33 drives protective antiviral CD8+ T cell responses. Science 2012; 335: 984-989.

13 Monticelli LA, Sonnenberg GF, Abt MC, et al. Innate lymphoid cells promote lung tissue homeostasis following acute influenza virus infection. Nat Immunol 2011; 12: 1045-1054.

14 Villarreal DO, Weiner DB. IL-33 isoforms: their future as vaccine adjuvants? Expert Rev Vaccines 2015; 14: 489-492. 